\title{
Long-term cost-effectiveness of matrix-associated chondrocyte implantation in the German health care system: a discrete event simulation
}

\author{
Tobias Vogelmann ${ }^{1}$. Philip P. Roessler ${ }^{2}$. Matthias Buhs ${ }^{3}$. Sven Ostermeier ${ }^{4}$ Justus Gille ${ }^{5}$. Arnd Hoburg ${ }^{6}$. \\ York Zöllner $^{7} \cdot$ Sebastian Schwarz $^{8} \cdot$ Tino Schubert $^{1} \cdot$ Marco Grebe $^{8} \cdot$ Wolfgang Zinser $^{9}$
}

Received: 29 September 2021 / Accepted: 14 December 2021 / Published online: 22 January 2022

(c) The Author(s) 2021

\begin{abstract}
Introduction Cartilage defects in the knee can be caused by injury, various types of arthritis, or degeneration. As a long-term consequence of cartilage defects, osteoarthritis can develop over time, often leading to the need for a total knee replacement (TKR). The treatment alternatives of chondral defects include, among others, microfracture, and matrix-associated autologous chondrocyte implantation (M-ACI). The purpose of this study was to determine cost-effectiveness of M-ACI in Germany with available mid- and long-term outcome data, with special focus on the avoidance of TKR.

Materials and methods We developed a discrete-event simulation (DES) that follows up individuals with cartilage defects of the knee over their lifetimes. The DES was conducted with a status-quo scenario in which M-ACI is available and a comparison scenario with no M-ACI available. The model included 10,000 patients with articular cartilage defects. We assumed Weibull distributions for short- and long-term effects for implant failures. Model outcomes were costs, number of TKRs, and quality-adjusted life years (QALYs). All analyses were performed from the perspective of the German statutory health insurance.

Results The majority of patients was under 45 years old, with defect sizes between 2 and $7 \mathrm{~cm}^{2}$ (mean: $4.5 \mathrm{~cm}^{2}$ ); average modeled lifetime was 48 years. In the scenario without M-ACI, $26.4 \%$ of patients required a TKR over their lifetime. In the M-ACI scenario, this was the case in only 5.5\% of cases. Thus, in the modeled cohort of 10,000 patients, 2700 TKRs, including revisions, could be avoided. Patients treated with M-ACI experienced improved quality of life (22.53 vs. 21.21 QALYs) at higher treatment-related costs (18,589 vs. 14,134 €/patient) compared to those treated without M-ACI, yielding an incremental cost-effectiveness ratio (ICER) of $3376 € /$ QALY.

Conclusion M-ACI is projected to be a highly cost-effective treatment for chondral defects of the knee in the German healthcare setting.
\end{abstract}

Keywords Chondral defects $\cdot$ Knee replacement $\cdot$ Cost-effectiveness $\cdot$ Discrete event simulation $\cdot$ Autologous chondrocyte implantation

Tobias Vogelmann and Philip P. Roessler have shared first authorship.

Tobias Vogelmann

tv@link-care.de

1 LinkCare GmbH, Kyffhäuserstr. 64, 70469 Stuttgart, Germany

2 Gelenkzentrum Mittelrhein GmbH, Mayen, Germany

3 Norddeutsches Knorpelcentrum, Quickborn, Germany

4 MVZ Gelenk-Klinik, Gundelfingen, Germany

5 University Hospital Schleswig-Holstein, Campus Luebeck, Luebeck, Germany
6 Med Center $360^{\circ}$, Berlin, Germany

7 Hamburg University of Applied Sciences, Hamburg, Germany

8 CO.DON AG, Leipzig, Germany

9 OrthoExpert Fohnsdorf, Austria and GFO-Kliniken Niederrhein, Dinslaken, Germany 


\section{Introduction}

Articular cartilage defects in the knee typically result from either degeneration or injuries. However, as articular cartilage is not supplied with blood, its ability to regenerate autonomously is limited and the defects are likely to progress to high-grade lesions if left untreated [1]. The most common symptoms of cartilage defects are pain, locking of the knee, and swelling. In the light of the latter, and under consideration of a high impact of articular cartilage defects on the quality of life [2] and productivity [3] of affected patients, the need for suitable treatment options is high. After a certain level of damage, conservative therapies are no longer sufficient, and surgery is unavoidable.

There are different treatment options available, in particular partial knee replacement (PKR), microfracture (MF), matrix-associated bone marrow stimulation (mBMS), autologous chondrocyte implantation (ACI), and matrix-induced autologous chondrocyte implantation (M-ACI). As a longterm consequence, osteoarthritis can develop over time and often leads to a need for total knee replacement (TKR) [4].

While the first generation of ACI consisted of chondrocytes being implanted under a periosteal flap and the second generation meant replacing this flap by a collagenous membrane, the third generation (M-ACI) is based on chondrocytes being seeded in a collagenous matrix. The latter is a fully autologous technique showing superior clinical results as compared to other methods, especially in larger lesions and regarding long-term outcomes [1,5-8]. To the best of our knowledge, there are limited data available on long-term economic effects comparing PKR, microfracture, mBMS, and M-ACI. Yet, due to ethical considerations, aspects of practicability, and the time delay, the evaluation of longterm real-world data is not warranted. The aim of this study was, therefore, to evaluate the cost-effectiveness of M-ACI in Germany for treatment of articular cartilage defects in the knee using available data. We hypothesize that the longer the need for a TKR is deferred to the future, the higher the discounted net economic benefit.

\section{Methods}

\section{Discrete event simulation model}

This study used a discrete event simulation (DES) model to compare long-term economic consequences of M-ACI compared to other available treatments from a statutory health insurance (SHI) perspective.

DES is a form of computer-based modeling methodology which is characterized by the ability to simulate dynamic behaviors of complex systems and interactions [9]; it has a long history as a major tool for operational research and gains increasing application in the healthcare sector [10].

The goal of DES is to compare multiple treatment options to identify the most efficient ones and has compared with other modeling systems without interaction, the advantage to being able to accommodate complexities at the individual level instead of the overall cohort level [11]. DES can simulate the temporal sequence of events in cases where a direct measurement is not applicable due to time delays, practicability, or ethical considerations. Within this model, patients are considered as independent entities which are linked to adjunct information, such as age, sex, and morbidity characteristics [12].

\section{Model structure and study population}

Table 1 and Figure S1 in the Supplemental material provide a schematic overview of the data sources and model architecture, respectively. Any individual enters the model with a symptomatic articular defect. Patient-level information about sex, age, and defect size of all patients treated with an M-ACI manufactured by CO.DON (chondrosphere ${ }^{\circledR}$ or Spherox ${ }^{\circledR}$ ) between 2007 and 2021 were provided by CO.DON AG, Leipzig, Germany. Based on a random sample of these data $(N=10,000)$, sex, age, and defect size of the articular defect were assigned to each modeled individual. The model used a time horizon until death for each individual, during which time patients would die in line with the official German life tables [13]. The symptomatic individual received nonsurgical therapies, such as analgesia, nonsteroidal anti-inflammatory drugs, land-based exercise, and others, until a surgical option became available. Once the decision for surgical intervention was made, the individual would receive the surgery according to their defect size (see "Interventions" below for details). This primary intervention may either be successful, i.e., the individual won't suffer any symptoms and the defect closes to $0 \mathrm{~cm}^{2}$, or unsuccessful. Unsuccessful interventions will keep the individual in a symptomatic health state until a successful intervention takes place. After a successful surgery of the defect, the implant will hold for a certain time and keep the individual symptomless until an event brings back a symptomatic defect. The process of surgery and implant failure will repeat for each individual until death. The model was run in two different healthcare scenarios: in the first scenario, matrix-induced autologous chondrocyte implantation is used in all patients as primary repair. This scenario is following the healthcare reality, since all patients entering the model were actually treated with M-ACI between 2007 and 2021. In a second, counter-factual scenario, M-ACI would not have been available to patients and patients would have 
Table 1 Parameters and data sources

\begin{tabular}{|c|c|c|}
\hline Parameters & Value & Source of information \\
\hline \multicolumn{3}{|l|}{ Population inputs } \\
\hline Population & Patient individual age, sex, defect size & CO.DON AG production data (data on file) \\
\hline Mortality rate & Depending on age and sex & DESTATIS [13] \\
\hline \multicolumn{3}{|l|}{ M-ACI } \\
\hline Eligibility for defect sizes & $>2 \mathrm{~cm}^{2}$ & Clinical guideline [13] \\
\hline Failure rate at 2 years & $7 \%$ & {$[24,25]$} \\
\hline Long-term failure rate & - & Weibull estimation based on $[24,25]$ (see online appendix) \\
\hline Costs & $11,000 €$ & Administrative DRG data (I18A, I08G, ZE126) [26] \\
\hline \multicolumn{3}{|l|}{ Microfracture } \\
\hline Eligibility for defect sizes & $\leq 2 \mathrm{~cm}^{2}$ & Clinical guideline [15] \\
\hline Failure rate at 2 years & $39 \%$ & {$[27]$} \\
\hline Long-term failure rate & - & Weibull estimation based on [27] (see online appendix) \\
\hline Costs & $1500 €$ & $\begin{array}{l}\text { Administrative DRG (I18B) [26] and outpatient renumera- } \\
\text { tion data (EBM 31133/36133) }\end{array}$ \\
\hline \multicolumn{3}{|l|}{ mBMS } \\
\hline Eligibility for defect sizes & $0-5 \mathrm{~cm}^{2}$ & Clinical guideline [15] \\
\hline Failure rate at 2 years & $4 \%$ & {$[28,29]$} \\
\hline Long-term failure rate & - & Weibull estimation based on $[28,29]$ (see online appendix) \\
\hline Costs & $3500 €$ & Administrative DRG data (I18A) [26] \\
\hline \multicolumn{3}{|l|}{ Partial knee replacement } \\
\hline Eligibility for defect sizes & $>10 \mathrm{~cm}^{2}$ & Expert opinion \\
\hline Failure rate at 2 years & $4 \%$ & {$[20]$} \\
\hline Long-term failure rate & - & Weibull estimation based on [20] (see online appendix) \\
\hline Costs & $6000 €$ & Administrative DRG data (I44E) [26] \\
\hline \multicolumn{3}{|l|}{ Total knee replacement } \\
\hline Eligible for defect sizes & $>10 \mathrm{~cm}^{2}$ & Expert opinion \\
\hline Failure rate at 2 years & $4 \%$ & {$[30]$} \\
\hline Eligible age for TKR & $55-80$ years & Assumption \\
\hline Long-term failure rate & - & Weibull estimation based on $[30,31]$ (see online appendix) \\
\hline Costs & $9000 €$ & Administrative DRG data (I44C) [26] \\
\hline Mortality rate at surgery & Depending on age and sex, $0.0449-0.11 \%$ & Clinical studies and registry data $[32,33]$ \\
\hline
\end{tabular}

been treated with other surgical or nonsurgical methods. All effectiveness and cost outcomes were computed by comparing the results from both scenarios which were run 10,000 times (once per patient) in both scenarios.

\section{Interventions}

\section{Microfracture}

In microfracturing, a small bone defect is caused to trigger the repair mechanisms in a joint with cartilage damage. It is used primarily for deep cartilage damage that extends to the underlying bone. During the procedure, the cortical bone is perforated, or small holes are drilled. Blood leaking from the wound flushes stem cells to the surface, which adheres to the bone. Differentiation of these stem cells induces the formation of new cartilage tissue. Microfracture is usually performed during arthroscopy and is performed in twothirds of cases on an outpatient basis and in one-third of the cases in an inpatient setting in Germany [14]. Symptomatic patients with defect sizes up to $2.0 \mathrm{~cm}^{2}$ are eligible for treatment with microfracture according to German treatment guidelines [15].

\section{mBMS}

Matrix-associated bone marrow stimulation (mBMS) is a modification of microfracture. After an arthroscopic assessment of the cartilage damage, an arthrotomy or arthroscopy is performed, and the shape and size are precisely measured. After that the subchondral bone is perforated and the matrix/ membrane in the correct size is applied and fixed with sutures or fibrin glue. The post-treatment regimen depends on the localization of the cartilage damage. The average 
length of stay in the hospital is around two to three days, followed by rehabilitation. Patients need to go on crutches for about 6 weeks [16]. The SHI covers the costs of mBMS, which is usually performed in the inpatient setting.

\section{$\mathrm{M}-\mathrm{ACl}$}

Matrix-induced autologous chondrocyte implantation (M-ACI) is a further development of the ACI procedure and is also suitable for severe cartilage damage. In an initial arthroscopic procedure, the patient's own healthy cartilage is extracted. In special laboratories, cells are isolated from cartilage samples, placed in culture media (depending on the manufacturer) and propagated, and then implanted into matrices or transferred to three-dimensional chondrospheres. In a second surgical procedure, this matrix-ACI-product is transplanted back into the knee. In the following weeks and months, the cartilage cells continue to produce new cartilage tissue. Maturation of this tissue takes about 12 months. The post-treatment regimen; thus, depends on the localization of the treated cartilage damage. The average length of stay (LoS) in hospital is about two to three days, followed by rehabilitation. The German SHI pays for the costs based on two inpatient treatments as covered by DRG (Diagnosis Related Groups) system, plus an additional fee ('Zusatzentgelt'). Symptomatic patients with defect sizes from $2.0 \mathrm{~cm}^{2}$ upwards are eligible for treatment with M-ACI according to German treatment guidelines.

\section{Partial and total knee replacement}

Knee replacement is one of the most common elective routine procedures in orthopaedic and trauma surgery in Germany, and worldwide, to treat patients with advanced osteoarthritis of the knee $[17,18]$. The following main criteria should be present for the indication of TKR: (i) knee pain for at least 3-6 months, (ii) evidence of structural damage (osteoarthritis, osteonecrosis) proven by X-ray, (iii) unsuccessful conservative therapeutic measures for at least 3-6 months, and (iv) limitation of quality of life related to the knee joint disease. For the indication for TKR, there should be evidence of structural damage (osteoarthritis, osteonecrosis) [19]. The intervention is fully covered by the SHI system. The surgery itself lasts around 60-90 min, the average LoS in hospital is seven days, and after hospital discharge, patients undergo a three-week rehabilitation. Patients need to go on crutches for about four weeks to three months and will be unable to work (sick leave) for around three to six months, depending on the physical demands of their job. The average service life of a TKR is assumed to be around 25 years [20]. However, approximately $10-20 \%$ of patients are not or not completely satisfied with the outcome after the surgery [21, 22], which is taken into account by quality-oflife decrements in the model.

\section{Modeling failure rates}

The information on failure rates was obtained from followup studies in the international literature (see Table 1). We assumed separate Weibull distributions for short- and longterm causes for implant failures. The probability-density curves of the two Weibull distributions were then merged to a joint distribution. Weibull models were adjusted to fit the observed values by means of the Lifedata. MLE function of the SPREDA package in R 4.0.3, the free software [23]. Detailed information is provided in the supplementary material.

\section{Health outcomes and costs}

Health gains from the interventions were expressed as QALYs and were calculated using the following technique: Between two subsequent events, individuals would either stay in the health state of symptomatic defects or in the state without symptomatic defects; patients would gain QALYs for every year in the corresponding state until a new event occurred. QALY weights were taken from a previous assessment of an M-ACI technology by NICE [4], see Table 2.

According to the German DRG catalogue and the billing catalogue for outpatient procedures ("Einheitlicher Bewertungsmaßstab", EBM), the procedure costs for an M-ACI were $11,000 €$, for an mBMS $3500 €$, for a microfracture $500 €$, for a TKR around $9000 €$, and a PKR around $6000 €$ (Table 1). A revision of a TKR was assumed to be $15,000 €$. Conservative therapy for a knee defect, e.g. physiotherapy and pain medication, was estimated to be $800 €$ per year.

\section{Perspective and discount rates}

The model was designed from the perspective of the German SHI system. Therefore, direct healthcare costs were considered, but no costs of working disability or early retirement were included in the model. Medical rehabilitation is partly funded by the pension insurance in Germany and was not considered. QALYs and costs were discounted a $2 \%$ discount rate in the base case scenario. As there is no official willingness to pay (WTP) threshold per QALY gained in Germany, we calculated a cost-effectiveness acceptability curve (CEAC), which shows the probability of M-ACI being a cost-effective option for different WTP thresholds.

\section{Accounting for uncertainty}

Uncertainty arises in the model in two ways: first, stochastic uncertainty and heterogeneity arising from structural 
Table 2 Health gains of the different interventions

\begin{tabular}{ll}
\hline Health state & $\begin{array}{l}\text { QALY weights } \\
\text { resp. gains per } \\
\text { year }\end{array}$ \\
\hline Before primary repair & 0.65 \\
Primary ACI, year 1 & 0.76 \\
Primary ACI, years 2+ & 0.82 \\
Primary MF, year 1 & 0.76 \\
Primary MF, years 2-4 & 0.82 \\
Primary MF, year 5 & 0.65 \\
Before 2nd repair & 0.65 \\
No 2nd repair & 0.69 \\
2nd repair ACI | 1st repair ACI, yr 1 & 0.76 \\
2nd repair ACI | 1st repair ACI, yr 2+ & 0.82 \\
2nd repair MF I 1st repair ACI, yr 1 & 0.76 \\
2nd repair MF | 1st repair ACI, yr 2-4 & 0.82 \\
2nd repair MF I 1st repair ACI, yr 5 & 0.65 \\
2nd repair MF I 1st repair MF, yr 1 & 0.76 \\
2nd repair MF I 1st repair MF, yr 2-4 & 0.82 \\
2nd repair MF I 1st repair MF, yr 5 & 0.65 \\
No further repair & 0.69 \\
Before 1st KR & 0.62 \\
Successful 1st KR I TKR/PKR & 0.78 \\
Before 2nd KR & 0.56 \\
Successful 2nd KR & 0.78 \\
No further KR & 0.69 \\
\hline
\end{tabular}

$A C I$ autologous chondrocyte implantation, $M F$ microfracture, $K R$ Knee replacement, TKR total knee replacement, $P K R$ partial knee replacement

or random variability in outcomes between patients. This uncertainty is typically accounted for in the context of Markov models with probabilistic sensitivity analyses (for example, Monte Carlo simulations). In the discrete event simulation used here, this uncertainty is already considered in the base case by 10,000 repetitions and the consideration of different individuals.

Secondly, parameter uncertainty arises. We have taken this into account by means of univariate sensitivity analyses, in which we have considered the influence of changes in assumptions on the outcomes. An one-way sensitivity analysis was performed and presented as tornado plot to assess the impact of a fixed change of $\pm 20 \%$ in input parameters on the ICER per QALY gained in the M-ACI scenario. As significant health gains and costs occur several decades after the initial surgery, especially costly TKR, and no official recommendation on discount rates in healthcare evaluations in the German system are present, we further assessed the impact of a discount rate of $4.5 \%$ for effects and costs. Also, since microfracture is commonly used in larger defect sizes [34], we also assessed a scenario where patients are eligible for microfracture for defect sizes as large as $5 \mathrm{~cm}^{2}$.
Table 3 Baseline characteristics in the discrete event simulation

\begin{tabular}{llll}
\hline & Total & Male & Female \\
\hline$N$ & 10,000 & $6069(60.7 \%)$ & $3931(39.3 \%)$ \\
Age in years & $36.1 \pm 11.0$ & $35.9 \pm 10.9$ & $36.6 \pm 11.3$ \\
$<35$ & $4531(45.3 \%)$ & $2833(46.7 \%)$ & $1698(43.2 \%)$ \\
$35-<45$ & $2955(29.5 \%)$ & $1823(30.0 \%)$ & $1132(28.8 \%)$ \\
$45-<55$ & $2245(22.5 \%)$ & $1258(20.7 \%)$ & $987(25.1 \%)$ \\
$\geq 55$ & $269(2.7 \%)$ & $155(2.6 \%)$ & $114(2.9 \%)$ \\
Defect size in $\mathrm{cm}^{2}$ & $4.4 \pm 2.6$ & $4.5 \pm 2.7$ & $4.1 \pm 2.6$ \\
$<2$ & $1360(13.6 \%)$ & $746(12.3 \%)$ & $614(15.6 \%)$ \\
$2-<4$ & $3477(34.8 \%)$ & $2030(33.4 \%)$ & $1447(36.8 \%)$ \\
$4-<6$ & $2641(26.4 \%)$ & $1652(27.2 \%)$ & $989(25.2 \%)$ \\
$6-<8$ & $1160(11.6 \%)$ & $745(12.3 \%)$ & $415(10.6 \%)$ \\
$8-<10$ & $982(9.8 \%)$ & $644(10.6 \%)$ & $338(8.6 \%)$ \\
$\geq 10$ & $380(3.8 \%)$ & $252(4.2 \%)$ & $128(3.2 \%)$ \\
\hline
\end{tabular}

Values are mean \pm standard deviation or number of patients $(\%)$. Percentage refers to column total, except percentage of $N$, which refers to line total

\section{Model development and statistical analysis}

The model was built in TreeAge Pro 2021, R1.2 Healthcare [35]. All input variables sourced from the literature were discussed among, and consented within, the author group, consisting of scientific and clinical experts in the treatment of chondral defects.

\section{Results}

\section{Baseline characteristics}

Upon entering the model, patients were 36 years old on average; approximately $60 \%$ were male; there was no significant age difference between men and women. Mean initial defect size was $4.2 \mathrm{~cm}^{2} .48 .4 \%$ of patients had initial defect sizes smaller than $4 \mathrm{~cm}^{2}$ (see Table 3). Median life expectancy was 84 years, so that the DES covered a median time horizon of 48 years.

\section{Number of interventions and total knee replacements}

Regarding the scenario with M-ACI use, a patient had his/ her first intervention at the age of 36 years. $82 \%$ of the patients had a second intervention, the mean time to $2^{\text {nd }}$ intervention being 17 years. Of those, 33\% renewed the $\mathrm{M}-\mathrm{ACI}, 63 \%$ had other surgical procedures, and $4 \%$ underwent TKR. $60 \%$ of these patients underwent a third intervention, this mean time accounting for another 6.6 years. Until death, a total of 5.5\% received a TKR at the average age of 65 years, 29 years after the first M-ACI. 13\% of the patients underwent a revision surgery after a mean of 
6 years after TKR. Until their death at the average age of 84 years, patients underwent 2.2 of the defined surgeries.

In the scenario without M-ACI, $35 \%$ of the patients underwent mBMS as primary intervention, while $24 \%$ underwent microfracture and 39\% received other procedures, such as PKR. As in the scenario with M-ACI, patients had their first intervention at the age of 36 years and an average defect size of $4.2 \mathrm{~cm}^{2} .86 \%$ of the patients underwent a second intervention after on average 12 years. Of those, 39\% underwent mBMS, $24 \%$ each received a microfracture or other surgical procedures, and $13 \%$ had a TKR. $66 \%$ of these patients underwent a third intervention after another 6.4 years. Until death, a total of $26 \%$ received a TKR at the average age of 62 years, 26 years after the initial intervention, and had, on average, three previous surgeries. $17 \%$ of patients who received a TKR underwent a revision surgery after a mean of 6 years after TKR. Until their death at the average age of 84 years, patients underwent 3.2 of the defined surgeries.

Observing the key figure of second surgeries, the percentage of patients with a second intervention increases over the years in both scenarios. At any time, the percentage of patients with a second intervention is significantly higher in the scenario without M-ACI; e. g. 25 years after the initial intervention, $47 \%$ of the patients with $\mathrm{M}$-ACI underwent a second surgery compared to $77 \%$ of the patients without M-ACI. Regarding the prevention of TKR, it can be observed that more patients in the nonM-ACI scenario received a TKR during the observation period. Compared to the non-M-ACI patients, $21 \%$ of TKRs in the M-ACI scenario were prevented (see Fig. 1).

Regarding the number of surgeries before a TKR, it can be observed that more patients in the scenario without M-ACI have a TKR (5.5\% in the scenario with M-ACI vs. $26 \%$ without), corresponding to a difference of $21 \%$. When compared to the scenario with M-ACI, patients without
M-ACI are, on average, one year younger when receiving their TKR.

\section{Quality of life, costs, and cost-effectiveness}

Total undiscounted costs in the scenario with M-ACI were $23,410 €$ and 19,669 $€$ in the M-ACI and non-M-ACI scenario, respectively. The cost difference of $3741 €$ was mainly driven by the costs for M-ACI $(16,445 €)$ in the M-ACI scenario which were partly offset by savings of $6929 €$ for avoided TKR. In discounted cost terms, costs were 18,590€ in the M-ACI scenario and 14,134 $€$ in the scenario without M-ACI, yielding a difference of $4455 €$ (see Fig. 2).

Costs of the two scenarios converged notably during the follow-up period (see Supplemental Material Figure S2).

Average QALYs in the 36 years of follow-up were 22.53 in the M-ACI scenario and 21.21 in the scenario without $\mathrm{M}-\mathrm{ACI}$, resulting in an incremental QALY gain of 1.32 in favor of the M-ACI scenario. Discounted costs per QALY gained were therefore $3376 €$ (4455€ cost difference divided by 1.32 difference in QALYs). Assuming a conservative cost-effectiveness threshold of 20,000 $€$ per QALY gained, M-ACI would be deemed cost-effective in $81.9 \%$ of all cases; it was found to be dominant, i.e., featuring lower total costs and higher QALYs, in $27.1 \%$ of cases (2710 simulated patients) (Fig. 3).

M-ACI scenario is cost-effective in more than $50 \%$ of cases if willingness to pay per QALY for the German healthcare system exceeds $5000 €$ per QALY. If willingness to pay exceeds 20,000 $€$ per QALY gained, M-ACI is a cost-effective treatment alternative in more than $80 \%$ of cases (Fig. 4).

\section{Sensitivity of results}

The univariate sensitivity analysis shows robust results for the ICER per QALY (Fig. 5). M-ACI failure rates and costs for M-ACI procedure had the largest impact on the
Fig. 1 Percentage of patients with multiple interventions/ TKR with M-ACI and without M-ACI

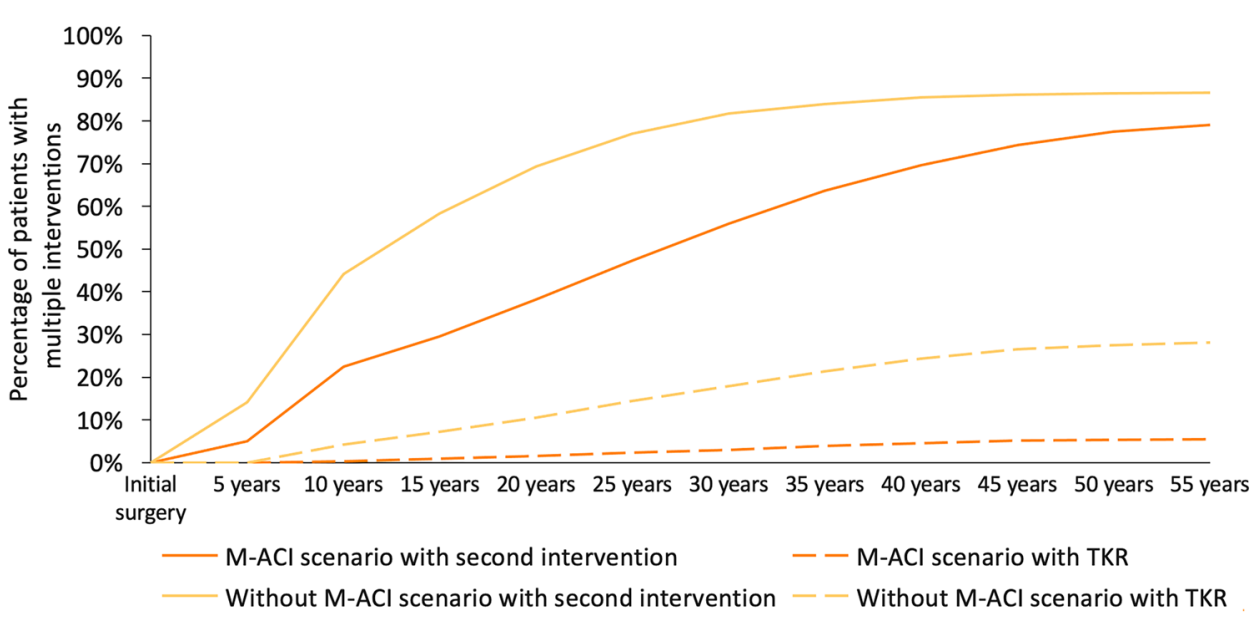


Fig. 2 a undiscounted and $\mathbf{b}$ discounted total costs by treatment scenario; isolated M-ACIonly and other intervention costs; M-ACI vs. non-M-ACI net cost difference encircled

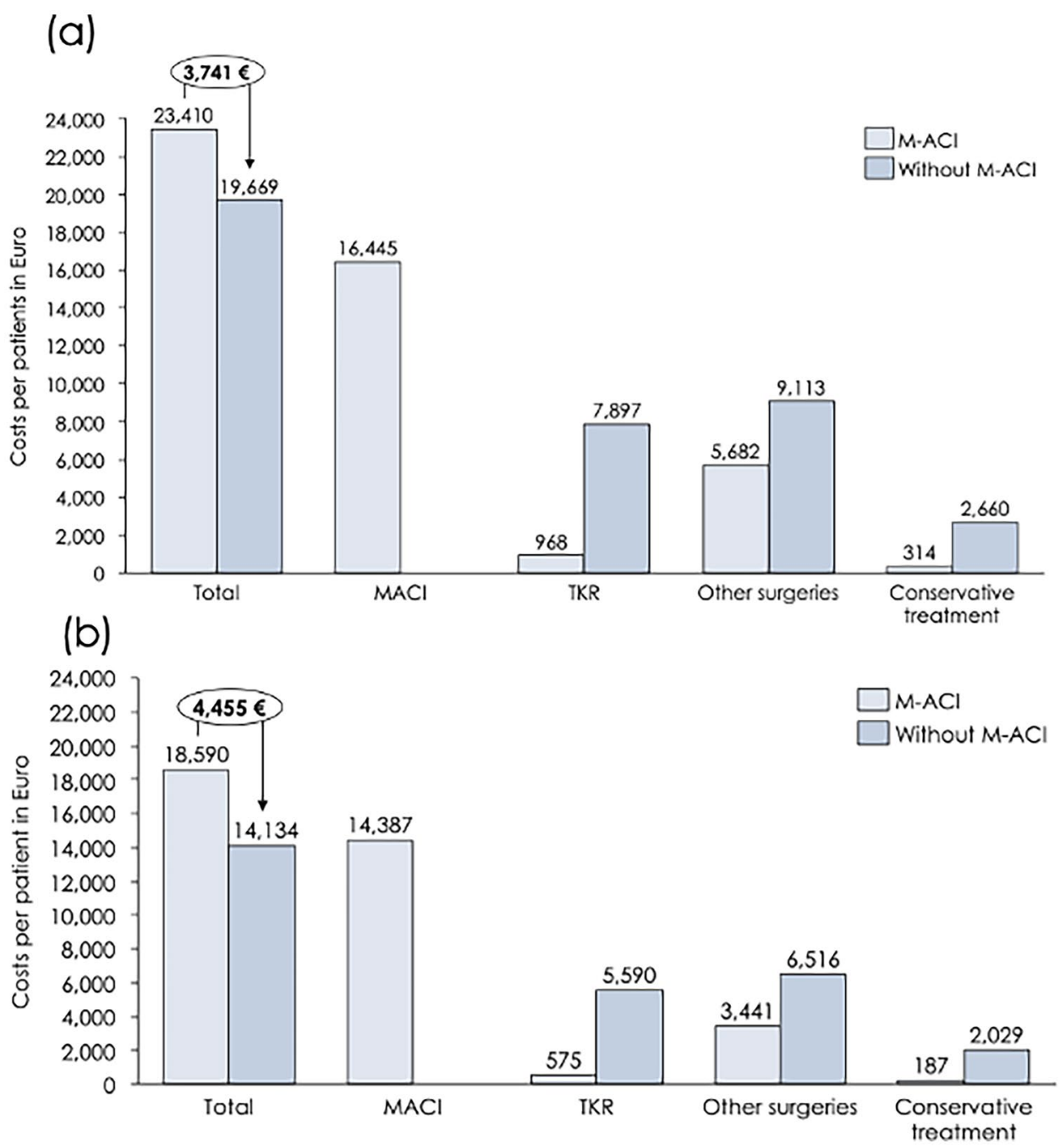

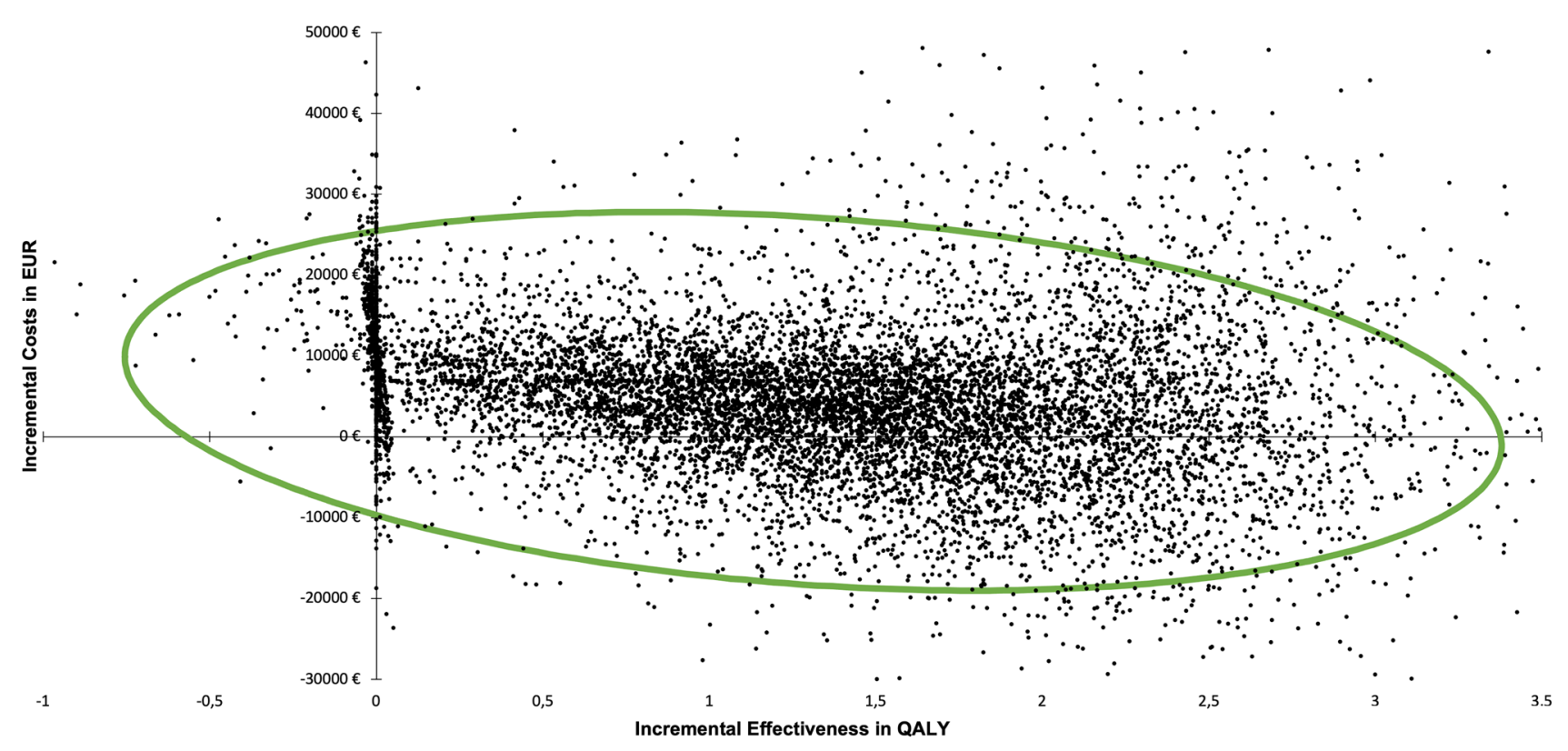

Fig. 3 Incremental cost-effectiveness of M-ACI vs. non-M-ACI scenarios 


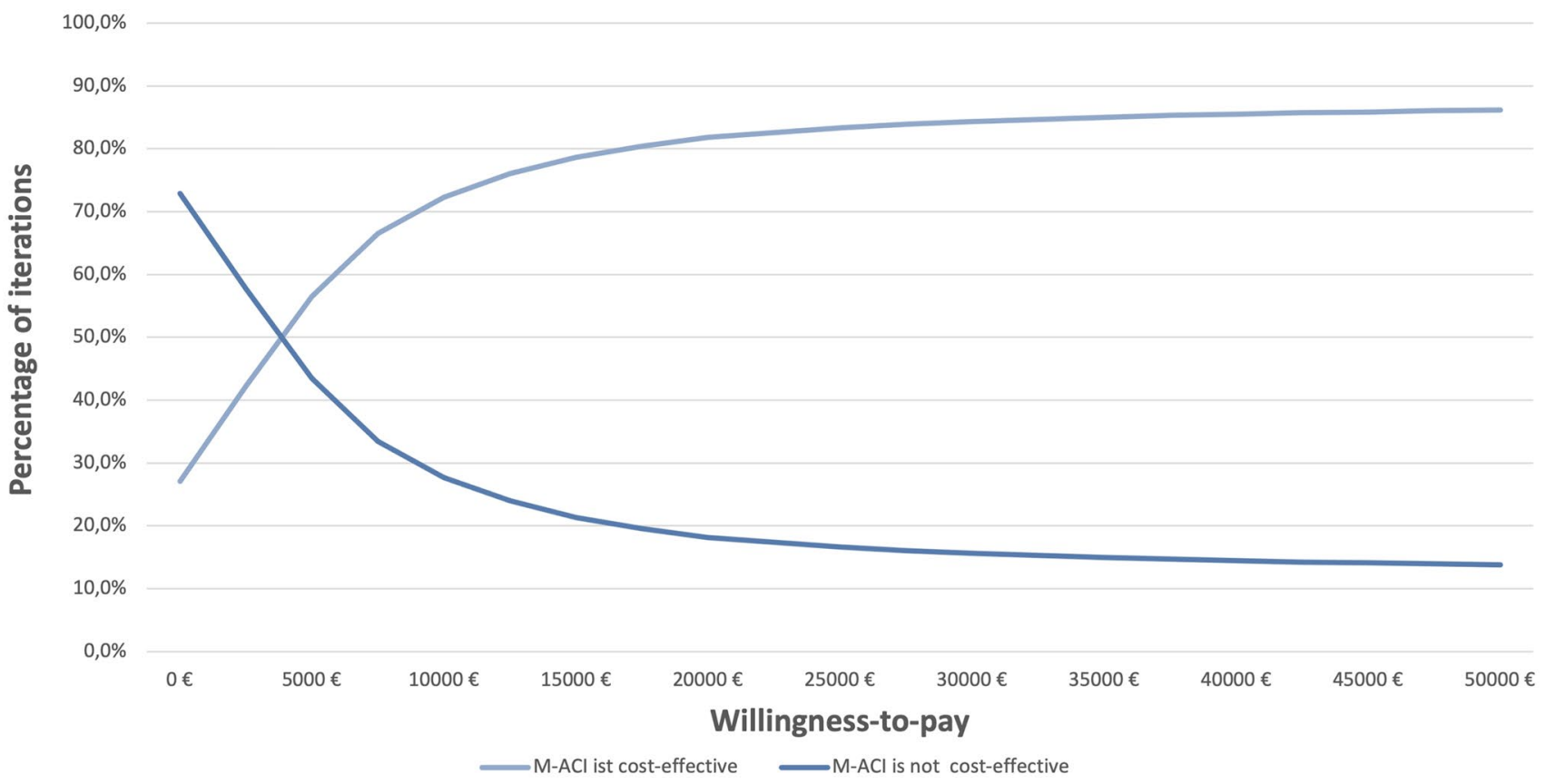

Fig. 4 Cost-effectiveness acceptability curve (CEAC) for treatment options. CEAC represent the probability for each scenario of being the most cost-effective option for different WTP thresholds. WTP is the maximum amount the healthcare system would be willing to pay

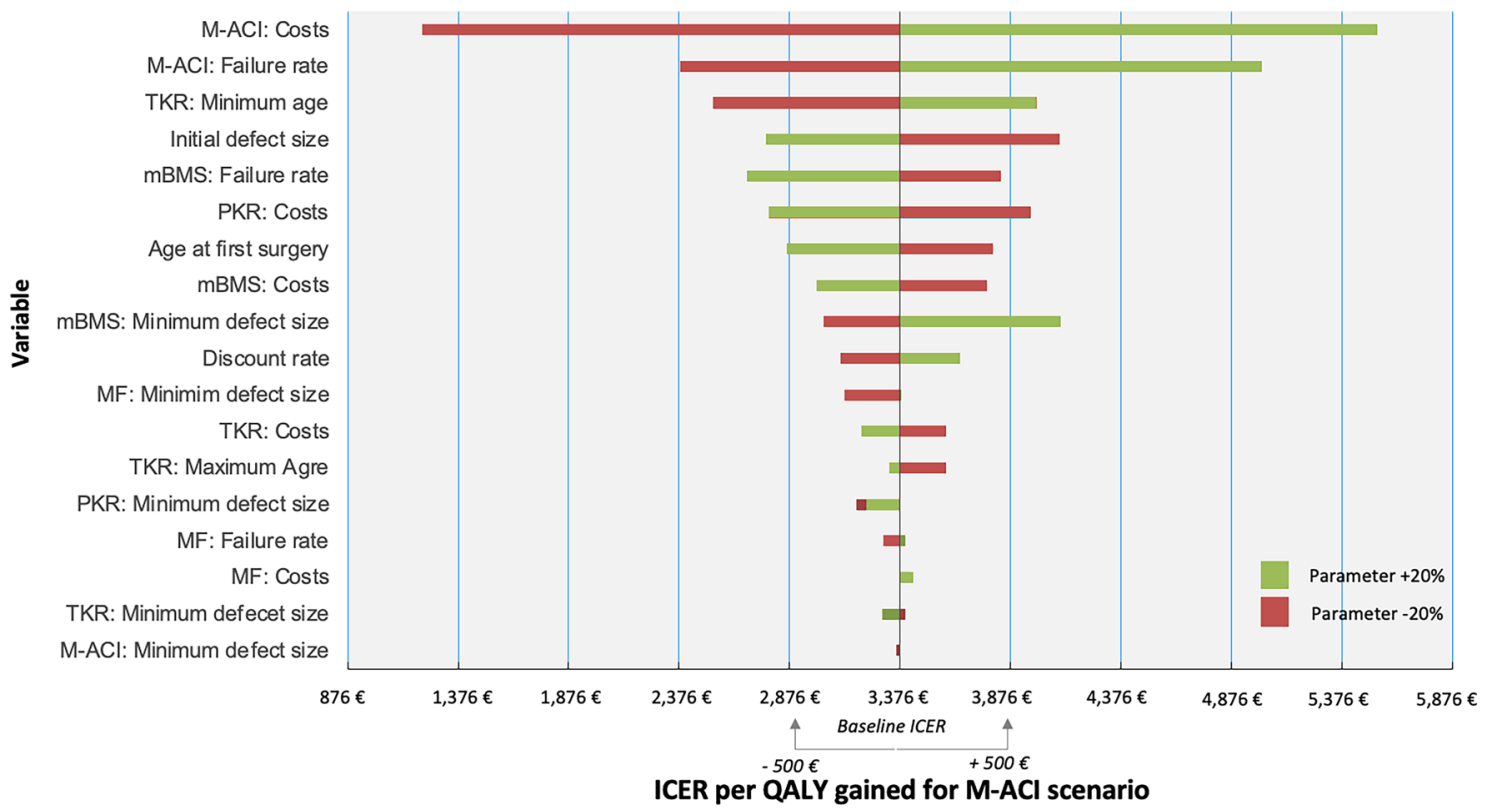

Fig. 5 Tornado plot showing the effect of the uncertainty surrounding the input parameters on the ICER per QALY gained for M-ACI scenario. Parameters were varied $\pm 20 \%$ from baseline scenario, the baseline values for the parameters used for this analysis can be found in Table 1

cost-effectiveness of this technology's application in the treatment process of cartilage defects in the knee. Within the range of $\pm 20 \%$ change, no parameter change led to an ICER per QALY gained of more than $6000 €$. The results were also robust for a change in discount rate to $4.5 \%$ per year with an ICER of $5348 €$ (Supplemental material, Table S1) and microfracture as a treatment option for larger defects with an ICER of $4872 €$ (Supplemental material, Table S2) per QALY gained. 


\section{Discussion}

In this modeling study, using patient-individual data on $10,000 \mathrm{M}-\mathrm{ACI}$ patients and a discrete event simulation approach, it was shown that $21 \%$ TKR can be avoided by using M-ACI in the overall population instead of individual other forms of knee cartilage repairs.

This drives quality of life of patients, who gain 1.32 QALY when operated with M-ACI as compared to a situation without the availability of M-ACI [36]. The increase in total lifetime costs is moderate, namely $€ 3741$ (undiscounted) viz. $€ 4455$ (discounted). At an ICER of $€ 3,376$ per QALY gained, the increase in lifetime quality of life would seem to justify the extra spending, as even a very strict willingness-to-pay (WTP) threshold, such as $€ 20,000 / \mathrm{QALY}$ gained (largely exhausted by many other means of treatment reimbursed under the German SHI scheme) would be undercut in over $80 \%$ of all cases. Already low willingness-to-pay thresholds as $10,000 €$ lead to M-ACI being cost-effective in more than $70 \%$ of cases. Furthermore, M-ACI showed as cost-effective in $50 \%$ of cases even if willingness to pay per QALY gained would be as low as $5000 €$. This high cost-effectiveness also showed as robust to one-way sensitivity analyses: Altering input parameters by plus/minus $20 \%$ did in no case results in ICERs exceeding $6000 €$ per QALY gained. Our model was most sensitive to altering the failure rate and costs of M-ACI. If the failure rate is $20 \%$ higher than in our base case, where we assumed $7 \%$ failure after 2 years, ICER per QALY gained would rise from 3376 to $5015 €$. Also, if costs of M-ACI would be $20 \%$ higher (i.e. $13,200 €$ instead of $11,000 €$ ), M-ACI would still be an cost effective treatment with an ICER per QALY gained of $5540 €$. No parameter change, including discount rates for costs and effects in this lifelong model and changes in indication for microfracture, did alter the results in a way that could question the cost-effectiveness of M-ACI.

Our results are well in line with the international literature, especially from Markov models in other European countries: In a NICE assessment [4], net QALY gains for M-ACI were between 0.0070 (18.189 QALY with M-ACI vs. 18.119 QALY without) and 2.410 (18.189 QALY vs. 15.779 QALY), with costs per incremental QALY of 4360 GBP (approx. $5100 €$ ).

Our study confirms previous estimates, based on German health claims data, namely an ICER per QALY gained over a lifetime lower than the GBP 4360 [14]. Our study also confirms recent works that found that $\mathrm{M}-\mathrm{ACI}$ is associated with significantly less re-operations, especially compared to microfracture, in the German healthcare setting [37].

Our analysis was based on a number of assumptions and has some limitations. First the quantification of the intervention effects was taken from different studies and not from head-to-head analyses. It cannot be excluded that patient characteristics differed between the different trials used to inform the model.

Another limitation was that the durability of the interventions was modeled from historical data, which may be underestimating the current survivorship of interventions given the technological advancement over decades. This issue has potential impact on cost-effectiveness of the different interventions, since an improved durability of would reduce the number of revisions. Furthermore, the model focussed on the treatment of the initial defect that might re-occur, but did not model new emerging defects explicitly, although new defects are included in the model implicitly as some re-interventions labeled as "revisions" in our model might be attributed to newly emerged defects.

Taken together, M-ACI is projected to be a highly costeffective treatment for chondral defects of the knee in the German healthcare setting. Further research is required to explore clinical long-term effects of cartilage repair and reduce uncertainty on long-term quality of life.

Supplementary Information The online version contains supplementary material available at https://doi.org/10.1007/s00402-021-04318-9.

Author contributions All authors contributed to the study conception and design. Material preparation, data collection, and analysis were performed by TV and TS. Data input and output validation were done by all authors, with PPR in leading role. The first draft of the manuscript was written by TV and all authors commented on previous versions of the manuscript. All authors read and approved the final manuscript.

Funding This study was financially supported by CO.DON AG, Leipzig, Germany.

Data availability No new data were created or analyzed in this study.

\section{Declarations}

Conflict of interest TV and TS hold shares and are employees of LinkCare $\mathrm{GmbH}$, which received consulting fees from CO.DON, TETEC and B.BRAUN in the last three years. PPR received research funding by Geistlich Biomaterials and Swissbiomed Orthopaedics. AH received consulting fees from CO.DON in the last three years. SO received consulting fees from Smith\&Nephew in the last three years. MG and SS are employees of CO.DON. The author authors declare no potential conflict of interest.

Ethical approval No new data were created or analyzed in this study. No ethics approval was required for this modeling study. This study was performed in line with the principles of the Declaration of Helsinki.

Informed consent No new data were created or analyzed in this study. 
Open Access This article is licensed under a Creative Commons Attribution 4.0 International License, which permits use, sharing, adaptation, distribution and reproduction in any medium or format, as long as you give appropriate credit to the original author(s) and the source, provide a link to the Creative Commons licence, and indicate if changes were made. The images or other third party material in this article are included in the article's Creative Commons licence, unless indicated otherwise in a credit line to the material. If material is not included in the article's Creative Commons licence and your intended use is not permitted by statutory regulation or exceeds the permitted use, you will need to obtain permission directly from the copyright holder. To view a copy of this licence, visit http://creativecommons.org/licenses/by/4.0/.

\section{References}

1. Hoburg A, Löer I, Körsmeier K et al (2019) Matrix-associated autologous chondrocyte implantation is an effective treatment at midterm follow-up in adolescents and young adults. Orthop J Sports Med 7:1-7. https://doi.org/10.1177/2325967119841077

2. Heir S, Nerhus TK, Røtterud JH et al (2010) Focal cartilage defects in the knee impair quality of life as much as severe osteoarthritis: a comparison of knee injury and osteoarthritis outcome score in 4 patient categories scheduled for knee surgery. Am J Sports Med 38:231-237. https://doi.org/10.1177/0363546509 352157

3. Lindahl A, Brittberg M, Peterson L (2001) Health economics benefits following autologous chondrocyte transplantation for patients with focal chondral lesions of the knee. Knee Surg Sports Traumatol Arthrosc 9:358-363. https://doi.org/10.1007/s001670100209

4. Armoiry X, Cummins E, Connock M et al (2019) Autologous chondrocyte implantation with chondrosphere for treating articular cartilage defects in the knee: an evidence review group perspective of a nice single technology appraisal. Pharmacoeconomics 37:879-886

5. Niemeyer P, Albrecht D, Andereya S et al (2016) Autologous chondrocyte implantation (ACI) for cartilage defects of the knee: a guideline by the working group "Clinical Tissue Regeneration" of the German Society of Orthopaedics and Trauma (DGOU). Knee 23:426-435. https://doi.org/10.1016/j.knee.2016.02.001

6. Liu YW, Tran MD, Skalski MR et al (2019) MR imaging of cartilage repair surgery of the knee. Clin Imaging 58:129-139

7. Riboh JC, Cvetanovich GL, Cole BJ, Yanke AB (2017) Comparative efficacy of cartilage repair procedures in the knee: a network meta-analysis. Knee Surg Sports Traumatol Arthrosc 25:37863799. https://doi.org/10.1007/s00167-016-4300-1

8. Salzmann GM, Paul J, Bauer JS et al (2009) T2 assessment and clinical outcome following autologous matrix-assisted chondrocyte and osteochondral autograft transplantation. Osteoarthritis Cartilage 17:1576-1582. https://doi.org/10.1016/j.joca.2009.07. 010

9. Pidd M (2006) Computer simulation in management science, 5th edn. John Wiley, New York

10. Taylor SJE, Robinson S (2006) So where to next? A survey of the future for discrete-event simulation. J Simulation 1:1-6

11. Brennan A, Chick SE, Davies R (2006) A taxonomy of model structures for economic evaluation of health technologies. Health Econ 15:1295-1310

12. Karnon J, Stahl J, Brennan A et al (2012) Modeling using discrete event simulation: a report of the ISPOR-SMDM modeling good research practices task force-4. Value in Health 15:821-827

13. Federal Statistical Office of Germany Sterbetafeln- Ergebnisse aus der laufenden Berechnung von Periodensterbetafeln für Deutschland und die Bundesländer
14. Niemeyer P, Schubert T, Grebe M, Hoburg A (2019) Treatment Costs of matrix-associated autologous chondrocyte implantation compared with microfracture: results of a matched-pair claims data analysis on the treatment of cartilage knee defects in Germany. Orthop J Sports Med 7:232596711988658

15. Niemeyer P, Becher C, Brucker PU et al (2018) Significance of matrix-augmented bone marrow stimulation for treatment of cartilage defects of the knee: a consensus statement of the DGOU Working Group on tissue regeneration. Z Orthop Unfall 156:e4-e4

16. Volz M, Schaumburger J, Frick $H$ et al (2017) A randomized controlled trial demonstrating sustained benefit of Autologous Matrix-Induced Chondrogenesis over microfracture at five years. Int Orthop 41:797-804

17. Carr AJ, Robertsson O, Graves S et al (2012) Knee replacement. The Lancet 379:1331-1340

18. Statistisches Bundesamt (2020) Fallpauschalenbezogene Krankenhausstatistik (DRG-Statistik). Operationen und Prozeduren der vollstationären Patientinnen und Patienten in Krankenhäusern

19. Lützner J, Lange T, Schmitt J et al (2018) S2k-Leitlinie: Indikation Knieendoprothese. Orthopade 47:777-781

20. Evans JT, Walker RW, Evans JP et al (2019) How long does a knee replacement last? A systematic review and meta-analysis of case series and national registry reports with more than 15 years of follow-up. The Lancet 393:655-663

21. Bourne RB, Chesworth BM, Davis AM et al (2010) Patient satisfaction after total knee arthroplasty: who is satisfied and who is not? Clin Orthop Relat Res 468:57-63

22. Dunbar MJ, Richardson G, Robertsson O (2013) I can't get no satisfaction after my total knee replacement. Bone Joint $\mathrm{J}$ 95-B:148-152

23. Hong Y, Xie Y, Xu Z (2018) Statistical package for reliability data analysis $\mathrm{v} 1.1$

24. Andriolo L, Reale D, Di Martino A et al (2020) Long-term results of arthroscopic matrix-assisted autologous chondrocyte transplantation: a prospective follow-up at 15 years. Am J Sports Med 48:2994-3001

25. Filardo G, Kon E, Andriolo L et al (2014) Clinical profiling in cartilage regeneration. Am J Sports Med 42:898-905

26. InEK Lump-sum reimbursement catalog 2021. https://www.g-drg. de/aG-DRG-System_2021/Fallpauschalen-Katalog/Fallpausch alen-Katalog_2021

27. Solheim E, Hegna J, Inderhaug E (2020) Long-term survival after microfracture and mosaicplasty for knee articular cartilage repair: a comparative study between two treatments cohorts. Cartilage 11:71-76. https://doi.org/10.1177/1947603518783482

28. Migliorini F, Eschweiler J, Maffulli N et al (2021) Autologous matrix-induced chondrogenesis (AMIC) and microfractures for focal chondral defects of the knee: a medium-term comparative study. Life 11:183

29. Migliorini F, Eschweiler J, Maffulli N et al (2021) Management of patellar chondral defects with autologous matrix induced chondrogenesis (AMIC) compared to microfractures: a four years followup clinical trial. Life 11:141

30. Grimberg A, Jansson V, Lützner J, et al EPRD annual report 2020

31. Rand JA, Ilstrup DM (1991) Survivorship analysis of total knee arthroplasty. Cumulative rates of survival of 9200 total knee arthroplasties. J Bone Joint Surg Am 73:397-409

32. IQTIG Knieendoprothesenversorgung - Qualitätsindikatoren und Kennzahlen: Bundesauswertung zum Erfassungsjahr 2019

33. Prange F, Seifert A, Piakong P et al (2021) Short-term mortality after primary and revision total joint arthroplasty: a singlecenter analysis of 103,560 patients. Arch Orthop Trauma Surg 141:517-525

34. Erggelet C, Vavken P (2016) Microfracture for the treatment of cartilage defects in the knee joint-a golden standard? J Clin 
Orthop Trauma 7:145-152. https://doi.org/10.1016/j.jcot.2016. 06.015

35. TreeAge Pro 2021 R1. TreeAge Software

36. NICE (2018) Single Technology Appraisal Autologous chondrocyte implantation with chondrosphere for treating articular cartilage defects [ID851]. 440

37. Niemeyer P, Schubert T, Grebe M, Hoburg A (2019) Matrix-associated chondrocyte implantation is associated with fewer reoperations than microfracture: results of a population-representative, matched-pair claims data analysis for cartilage defects of the knee. Orthop J Sports Med 7:232596711987784

Publisher's Note Springer Nature remains neutral with regard to jurisdictional claims in published maps and institutional affiliations. 